\title{
Dehydroepiandrosterone Sulfate as a Risk Factor for Premature Myocardial Infarction: A Comparative Study

\author{
Mohammad Shojaie ${ }^{1}$, Mohammad Yaghoub Rajpout, Armin Abtahian ${ }^{2}$, \\ Azadeh Esmail Pour ${ }^{3}$, Mohamed Amin Ghobadifar ${ }^{2, *}$, Armin Akbarzadeh ${ }^{4}$
}

Department of Internal Medicine, ${ }^{1}$ Research Center for Social Determinants of Health, and ${ }^{2}$ Department of Student Research Committee, Jahrom University of Medical Sciences; ${ }^{3}$ Cardiac Care Unit Nurse, Peymanieh Hospital, Jahrom University of Medical Sciences, Jahrom; ${ }^{4}$ Student Research Committee, Shiraz University of Medical Sciences, Shiraz, Iran

\section{ABSTRACT}

Background: This study aimed to evaluate some of the major risk factors of myocardial infarction including dehydroepiandrosterone sulfate in patients with premature myocardial infarction (age $<50$ years old) and myocardial infarction (age $\geq 50$ years).

Methods: This is a parallel case-control study on 50 premature myocardial infarction patients and 50 myocardial infarction patients. We also recruited 50 matched participants for each of the two groups. Patients and their control groups were assessed for dehydroepiandrosterone sulfate serum level, diabetes mellitus, hyperlipidemia, hypertriglyceridemia, and hypertension. In addition, family history of cardiovascular disease and current smoking was recorded. Univariate and multivariate logistic regression analyses were performed to evaluate predictors of premature myocardial infarction and myocardial infarction.

Results: No significant differences were observed between the demographic data of patients and their controls. The dehydroepiandrosterone sulfate serum level was significantly higher in patients with premature myocardial infarction compared with controls. Multivariate logistic regression analysis revealed only serum dehydroepiandrosterone sulfate dehydroepiandrosterone sulfate level to be significantly associated with premature myocardial infarction (odds ratio, 2.65; 95\% confidence interval, 1.44 to 4.877 ; $\mathrm{P}=0.002$ ). Additionally, hypertension was found to be associated with myocardial infarction.

Conclusion: Higher levels of serum dehydroepiandrosterone sulfate level are associated with premature myocardial infarction but not with myocardial infarction, and this association is independent of the effects of other risk factors.

Keywords: Dehydroepiandrosterone Sulfate; Myocardial Infarction; Risk Factors

\footnotetext{
Received: May 28, 2014, Accepted: November 10, 2014

*Corresponding Author: Mohamed Amin Ghobadifar

Tel: +98-936-620-8078, Fax: +98-711-636-13-86, E-mail: amin_m505@yahoo.com

Korean Journal of Family Medicine

Copyright (C) 2015 The Korean Academy of Family Medicine

This is an open-access article distributed under the terms of the Creative Commons Attribution Non-Commercial License (http://creativecommons.org/licenses/by-nc/3.0) which permits unrestricted noncommercial use, distribution, and reproduction in any medium, provided the original work is properly cited.
} 


\section{INTRODUCTION}

The incidence of myocardial infarction, the most common subtype of coronary artery disease, is on the rise in young adults. ${ }^{1)}$ Previous studies have revealed that approximately $18 \%$ of acute myocardial infarction patients are $<50$ years old, and this is referred to as premature myocardial infarction. ${ }^{2}$ These patients show a higher re-infarction rate, more occurrences of severe heart failure, and also a significantly higher mortality rate due to cardiovascular events at a young age. ${ }^{3)}$

It is believed that myocardial infarction risk factors, clinical features, and prognosis are different in young and older patients. ${ }^{4}$ Several investigations have shown that endogenous hormones play a remarkable etiological role in coronary artery disease. They can have a direct effect as an independent risk factor or an indirect effect via other coronary artery disease risk factors such as lipids and lipoproteins. ${ }^{5,6)}$ Unbalanced levels of gonadal hormones such as testosterone, estrogen, and their precursor, dehydroepiandrosterone sulfate are considered responsible for premature myocardial infarction.

Previous studies have provided conflicting results about the effect of dehydroepiandrosterone sulfate on coronary artery disease. These studies have revealed either an inverse, ${ }^{7-11)}$ null, ${ }^{12,13)}$ or positive ${ }^{14,15)}$ relationship between dehydroepiandrosterone sulfate levels and coronary artery disease. This comparative study investigates the role of dehydroepiandrosterone sulfate in both premature myocardial infarction and myocardial infarction of both sexes in the Iranian population.

\section{METHODS}

\section{Study Design}

The current study involved a comparative, descriptive, cross-sectional survey conducted in Peymanieh Hospital, affiliated with Jahrom University of Medical Sciences, from March 2011 to July 2012. We included 50 patients with premature myocardial infarction ( $<50$ years) and 50 patients with myocardial infarction ( $>50$ years). The myocardial infarction diagnosis was made based on two of the three World Health Organization criteria. ${ }^{16)}$ We also included 100 participants as controls: 50 people $<50$ years old (control 1) and 50 people $>50$ years old (control 2). Control subjects were frequency matched for sex and age in the design stage. In order to avoid over fitting, other variables were controlled through regression methods.

Both the patient groups were recruited from the Peymanieh Hospital in Jahrom, and the control groups were randomly selected from the healthy participants of our parent study. ${ }^{17)}$ Randomization was performed by a computer-based digital random generator. Of the healthy participants, only those with no history of coronary artery disease and no World Health Organization criterion for myocardial infarction were included in the analysis. According to the World Health Organization criteria, a cardiac troponin rise accompanied 
by either typical symptoms, pathological Q waves, ST elevation, or depression or coronary intervention are diagnostic of myocardial infarction. Patients and controls who had a history of endocrine conditions such as pituitary disease or adrenal gland disease and hormone replacement therapy, women with ovarian disease, and individuals reporting the use of any medications with the potential to alter lipid, lipoprotein, or hormone levels were excluded. Additionally, those with a history of cancer were excluded since dehydroepiandrosterone sulfate could have antiproliferative effects and also because lower levels have been implicated in the etiology of various kinds of cancers. ${ }^{9,18,19)}$ Information on these variables was obtained by interviewing patients and/or their family. The study protocol was approved by the review board and ethics committee of Jahrom University of Medical Sciences. Written informed consents were obtained from all recruited patients after they were fully informed about the study protocol.

\section{Study Protocol}

All patients underwent a complete history taking and a physical examination by a cardiologist. The information was recorded in a questionnaire consisting of age, sex, current smoking (smoking within 1 year), diabetes mellitus (symptoms of diabetes plus casual plasma glucose concentration $>200 \mathrm{mg} / \mathrm{dL}$, fasting plasma glucose concentration $>126 \mathrm{mg} / \mathrm{dL}$, 2-hour plasma glucose concentration $>200 \mathrm{mg} / \mathrm{dL}$ during $75 \mathrm{~g}$ oral glucose tolerance test, or receiving medication for diabetes mellitus) and hypertension (>140/90 mm Hg or receiving antihypertensives), family history of coronary artery disease (in firstdegree relative), and administration of any medication.

A peripheral venous blood sample was obtained at study initiation from all patients in the control groups and at the time of admission in those with myocardial infarction. Dehydroepiandrosterone sulfate concentrations were assessed by enzyme-linked immunosorbent assay (ELISA; DIA-PRO, Rome, Italy) according to the manufacturer's instruction from serum samples, which were stored at $-70^{\circ} \mathrm{C}$ for an average of 6 months. Another sample was obtained after 12 hours of fasting and sent to the laboratory to measure the serum total cholesterol, high density lipoprotein cholesterol, and fasting blood sugar using the enzymatic method. Hypercholesterolemia was considered as a fasting serum total cholesterol level of $240 \mathrm{mg} / \mathrm{dL}$ or higher or if the patient was being treated with lipid-lowering medication. All measurements were performed in the hospital facilities according to standardized protocols. The inter-assay and intra-assay coefficients of variation were performed $<6 \%$ for all measurements.

\section{Statistical Analyses}

Fifty patients were included in each group so that the power of study was $90 \%$ to detect significant differences between various variables. All statistical analyses were performed using the SPSS ver. 15.0 (SPSS Inc., Chicago, IL, USA). Chi-square test was employed to compare the categorical parameters between patients and their controls. Furthermore, t-test was employed to compare the continuous parameters. Univariate and multivariate logistic regression analyses were performed to evaluate predictors of premature myocardial infarction and myocardial infarction. $\mathrm{P}<0.05$ was considered statistically significant. 


\section{RESULTS}

Overall, 200 participants were included: 50 with premature myocardial infarction, 50 with myocardial infarction, and 50 participants as control group for each of the two patient groups. The mean age of the patients with premature myocardial infarction and control 1 group was $44.4 \pm 3.8$ and $45.5 \pm 2.5$ years, respectively; it was $58.0 \pm 5.8$ and $56.0 \pm 4.4$ years for the myocardial infarction patients and control 2 group, respectively. Baseline characteristics and lipid profile of each group are shown in Table 1. According to Table 2, the mean ages are not significantly different between patients and their control groups. Moreover, it reveals no differences between premature myocardial infarction and myocardial infarction patients and their control groups regarding risk factors. In a similar manner, myocardial infarction patients had no significant differences with their control group and with premature myocardial infarction patients in the risk factors. Dehydroepiandrosterone sulfate level was significantly higher in premature myocardial infarction patients compared with control 1 group $(P=0.002)$, while myocardial infarction patients did not have significant differences with control 2 group $(\mathrm{P}=0.256)$ (Figure 1). Furthermore, the dehydroepiandrosterone sulfate level was higher in premature myocardial infarction patients compared with myocardial infarction patients $(\mathrm{P}<0.001)$. On the other hand, the difference between male and female premature myocardial infarction patients was not significant $(\mathrm{P}=$ 0.095). In univariate and multivariate logistic regression analysis, dehydroepiandrosterone sulfate level was found to be an independent predictor of premature myocardial infarction (Table 2).

Table 1. Baseline characteristics and lipid profile of patients with premature myocardial infarction ( $<50 \mathrm{y}$ of age) and $\mathrm{MI}(>50 \mathrm{y}$ of age)

\begin{tabular}{lcccccc}
\hline \multicolumn{1}{c}{ Characteristic } & Premature MI & Control 1 & P-value & MI patients & Control 2 & P-value \\
\hline Age (y) & $44.4 \pm 3.8$ & $45.5 \pm 2.5$ & 0.090 & $58.0 \pm 5.8$ & $56.0 \pm 4.4$ & 0.091 \\
Sex & & & & & & \\
$\quad$ Male & $6(72)$ & $34(68)$ & 0.663 & $29(58)$ & $33(66)$ & 0.410 \\
$\quad$ Female & $14(28)$ & $16(32)$ & & $21(42)$ & $17(34)$ & \\
Current smoking & $21(42)$ & $23(46)$ & 0.691 & $20(40)$ & $17(35)$ & 0.539 \\
Hypertension & $11(22)$ & $10(20)$ & 0.236 & $17(35)$ & $9(18)$ & 0.069 \\
DHEAS (ug/mL) & $1.67 \pm 0.85^{*}$ & $1.18 \pm 0.68$ & 0.002 & $0.89 \pm 0.88^{\dagger}$ & $1.04 \pm 0.68$ & 0.256 \\
Diabetes mellitus & $16(32)$ & $10(20)$ & 0.175 & $19(38)$ & $13(26)$ & 0.202 \\
Hypercholesterolemia & $13(26)$ & $14(28)$ & 0.82 & $13(26)$ & $16(32)$ & 0.513 \\
Low high density lipoprotein & $18(36)$ & $13(26)$ & 0.513 & $10(20)$ & $11(22)$ & 0.398 \\
Cardiovascular family history & $29(58)$ & $28(56)$ & 0.84 & $31(62)$ & $26(52)$ & 0.313 \\
\hline
\end{tabular}

Values are presented as mean $\pm \mathrm{SD}$ or number $(\%)$.

DHEAS: dehydroepiandrosterone sulfate, MI: myocardial infarction.

*Serum DHEAS level was not significantly different between men and women with premature MI $(\mathrm{P}=0.085)$. †Serum DHEAS level was significantly different between the premature MI and MI groups $(\mathrm{P}<0.05)$. 
Table 2. DHEAS level in premature MI and MI patients in univariate and multivariate logistic regression. The DHEAS serum level was significantly associated with premature MI

\begin{tabular}{lccccc}
\hline \multirow{2}{*}{ Variable } & \multicolumn{2}{c}{ Univariate analysis } & & \multicolumn{2}{c}{ Multivariate analysis } \\
\cline { 2 - 3 } \cline { 5 - 6 } & OR $(95 \% \mathrm{CI})$ & P-value & & OR $(95 \% \mathrm{CI})$ & P-value \\
\hline DHEAS serum level in premature MI & $2.282(1.316-3.958)$ & 0.003 & & $2.65(1.448-4.877)$ & 0.002 \\
DHEAS serum level in MI & $0.697(0.374-1.300)$ & 0.256 & & $0.680(0.332-1.393)$ & 0.292 \\
\hline
\end{tabular}

DHEAS: dehydroepiandrosterone sulfate, MI: myocardial infarction, OR: odds ratio, CI: confidence interval. *Variable included in the model: age, sex, current smoking, hypertension, diabetes mellitus, hypercholesterolemia, low high density lipoprotein, and family history of cardiovascular disease.

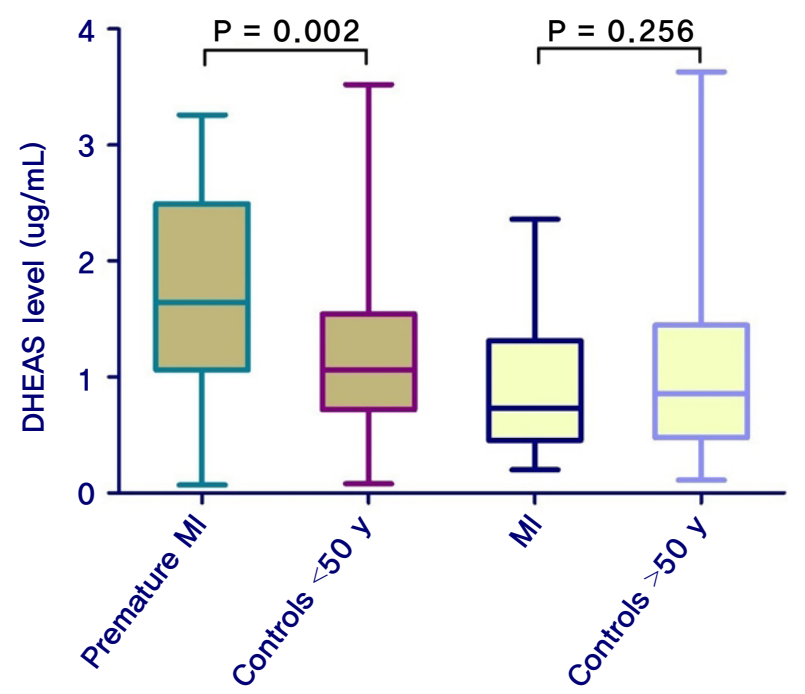

Figure 1. Median DHEAS level in each group of participants. T-test was employed to compare the DHEAS level between the cases and their controls. DHEAS level was significantly higher in patients with premature MI as compared with controls. DHEAS: dehydroepiandrosterone sulfate, MI: myocardial infarction.

\section{DISCUSSION}

Ischemic heart disease is the leading cause of mortality in high-income countries and the second leading cause in middle-income countries. ${ }^{20)}$ Understanding the pathogenesis and identifying its risk factors is important for prevention and effective treatment of this disease. The purpose of this matched case-control study was to investigate the plasma dehydroepiandrosterone sulfate levels in premature myocardial infarction (age $<50$ years) and myocardial infarction (age $>50$ years) patients. We found that there is a positive relationship between the dehydroepiandrosterone sulfate level and premature myocardial infarction but not between the dehydroepiandrosterone sulfate level and myocardial infarction. There is still controversy surrounding the role of dehydroepiandrosterone sulfate in myocardial infarction.

The only study conducted so far on a comparison of dehydroepiandrosterone sulfate levels in premature myocardial infarction was by Mitchell et al. ${ }^{21)}$ and involved only men. This study indicated 
an inverse relationship between serum dehydroepiandrosterone sulfate levels and premature myocardial infarction in men. Our study does not confirm that dehydroepiandrosterone sulfate level is always indicative of better health.

Several studies have revealed that low levels of dehydroepiandrosterone sulfate may be related to diseases such as cancer, osteoporosis, deterioration of lipid metabolism, and neurological problems. ${ }^{22-25}$ ) Some studies have provided evidence indicating that a low serum dehydroepiandrosterone sulfate level is associated with cardiovascular disease, whereas some others have shown no association between serum dehydroepiandrosterone sulfate level and cardiovascular disease. ${ }^{9,26,27)}$ Similar to the results of our study, some studies have indicated a positive association between dehydroepiandrosterone sulfate and cardiovascular disease, ${ }^{14,15)}$ However, these studies were performed only on women. Some believe that there is no association between dehydroepiandrosterone sulfate level and cardiovascular disease in women but that there is an inverse relation in men. However, our study shows a significantly higher dehydroepiandrosterone sulfate plasma level in premature myocardial infarction patients in both men and women. The dehydroepiandrosterone sulfate plasma level was also similar between men and women.

A study with results similar to ours, reported an odds ratio of 6.48 for the dehydroepiandrosterone sulfate level when it was justified for age. Unlike our results, this study, which was performed on middle-aged men, reported a risk association with dehydroepiandrosterone sulfate serum level in older patients but not with the younger patients. ${ }^{17)}$ Although our study revealed an odds ratio of 2.65 in premature myocardial infarction patients, when it was adjusted for other major risk factors, no risk was associated with high dehydroepiandrosterone sulfate levels in myocardial infarction patients. The initial analysis of the Rancho Bernardo Study ${ }^{28)}$ on 30 deaths from coronary artery disease, during a 12-year follow-up of postmenopausal women 60 to 79 years of age, indicated that although dehydroepiandrosterone sulfate levels were unrelated to the risk of fatal cardiovascular disease in women, they were associated with several major cardiovascular disease risk factors. A probable explanation was that a steroid biosynthetic defect of the adrenal glands or functional adrenal hyperplasia caused increased chronic heart disease risk, which is associated with high dehydroepiandrosterone sulfate levels. Our study shows that this higher levels of dehydroepiandrosterone sulfate are independent of some of the common risk factors of myocardial infarction, such as current smoking, hypertension, hypercholesterolemia, diabetes mellitus, hypertriglyceridemia, and family history of cardiovascular conditions (if any member of first relative family had coronary artery disease).

The reasons for the association between the dehydroepiandrosterone sulfate level and coronary artery disease are not clear. A cohort study on postmenopausal women demonstrated increased risk of myocardial infarction at higher testosterone levels. ${ }^{29)}$ Dehydroepiandrosterone sulfate is a precursor of sex hormones such as testosterone and estrogen. A possible explanation for the positive relationship between serum dehydroepiandrosterone sulfate level and premature myocardial infarction can be the effect of dehydroepiandrosterone sulfate via other sex hormones such as testosterone. On the other hand, dehydroepiandrosterone sulfate level was higher in premature myocardial infarction 
patients compared with myocardial infarction patients. This may be due to characteristics of dehydroepiandrosterone sulfate, the level of which declines with age. ${ }^{30)}$

We tried to exclude the role of confounding factors by adjusting for some of the major risk factors of myocardial infarction, using multivariate regression analysis. However, some factors such as body mass index, physical activity, alcohol use, low density lipoprotein cholesterol, triglycerides, C-reactive protein, and menopausal status were not included in the model.

Similar to other studies, our study also has limitations. However, this was the first comparative study performed on both premature myocardial infarction and myocardial infarction patients in both men and women. The result reveals that higher levels of serum dehydroepiandrosterone sulfate are associated with premature myocardial infarction but not with myocardial infarction and that this association is independent of the effects of other major risk factors.

\section{CONFLICT OF INTEREST}

No potential conflict of interest relevant to this article was reported.

\section{ACKNOWLEDGMENTS}

We would like to thank all the patients and their respectful families who participated in the present study. This study is the thesis of Dr. Abtahian, which was supported by the authorities of Jahrom University of Medical Sciences. The authors would like to thank Dr. Nasrin Shokrpour for editorial assistance and Mrs Sareh Roosta for statistical analysis at the Center for Development of Clinical Research of Nemazee Hospital. Additionally, authors would like to thank the Arshan group for editorial assistance.

\section{REFERENCES}

1. He J, Gu D, Wu X, Reynolds K, Duan X, Yao C, et al. Major causes of death among men and women in China. N Engl J Med 2005;353:1124-34.

2. Sarraf-Zadegan N, Sayed-Tabatabaei FA, Bashardoost N, Maleki A, Totonchi M, Habibi HR, et al. The prevalence of coronary artery disease in an urban population in Isfahan, Iran. Acta Cardiol 1999;54:257-63.

3. Fournier JA, Cabezon S, Cayuela A, Ballesteros SM, Cortacero JA, Diaz De La Llera LS. Long-term prognosis of patients having acute myocardial infarction when $<=40$ years of age. Am J Cardiol 2004;94:989-92.

4. Chen L, Chester M, Kaski JC. Clinical factors and angiographic features associated with premature coronary artery disease. Chest 1995;108:364-9. 
5. Crandall CJ, Barrett-Connor E. Endogenous sex steroid levels and cardiovascular disease in relation to the menopause: a systematic review. Endocrinol Metab Clin North Am 2013;42:227-53.

6. Xu L, Freeman G, Cowling BJ, Schooling CM. Testosterone therapy and cardiovascular events among men: a systematic review and meta-analysis of placebo-controlled randomized trials. BMC Med 2013;11:108.

7. Traish AM, Kang HP, Saad F, Guay AT. Dehydroepiandrosterone (DHEA): a precursor steroid or an active hormone in human physiology. J Sex Med 2011;8:2960-82.

8. Dumas de la Roque E, Savineau JP, Bonnet S. Dehydroepiandrosterone: a new treatment for vascular remodeling diseases including pulmonary arterial hypertension. Pharmacol Ther 2010;126:186-99.

9. Ohlsson C, Labrie F, Barrett-Connor E, Karlsson MK, Ljunggren O, Vandenput L, et al. Low serum levels of dehydroepiandrosterone sulfate predict all-cause and cardiovascular mortality in elderly Swedish men. J Clin Endocrinol Metab 2010;95:4406-14.

10. Bonnet S, Paulin R, Sutendra G, Dromparis P, Roy M, Watson KO, et al. Dehydroepiandrosterone reverses systemic vascular remodeling through the inhibition of the Akt/GSK3-\{beta\}/NFAT axis. Circulation 2009;120:1231-40.

11. Feldman HA, Johannes CB, Araujo AB, Mohr BA, Longcope C, McKinlay JB. Low dehydroepiandrosterone and ischemic heart disease in middle-aged men: prospective results from the Massachusetts Male Aging Study. Am J Epidemiol 2001;153:79-89.

12. Shibli-Rahhal A, Haynes W, Sinkey C, Dillon J. Effect of dehydroepiandrosterone (DHEA) on vascular function in postmenopausal women with diabetes: a randomized controlled trial. J Diabetes Res Clin Metab 2012;1:13.

13. Boxer RS, Kleppinger A, Brindisi J, Feinn R, Burleson JA, Kenny AM. Effects of dehydroepiandrosterone (DHEA) on cardiovascular risk factors in older women with frailty characteristics. Age Ageing 2010;39:451-8.

14. Page JH, Ma J, Rexrode KM, Rifai N, Manson JE, Hankinson SE. Plasma dehydroepiandrosterone and risk of myocardial infarction in women. Clin Chem 2008;54:1190-6.

15. Johannes CB, Stellato RK, Feldman HA, Longcope C, McKinlay JB. Relation of dehydroepiandrosterone and dehydroepiandrosterone sulfate with cardiovascular disease risk factors in women: longitudinal results from the Massachusetts Women's Health Study. J Clin Epidemiol 1999;52:95-103.

16. Mendis S, Thygesen K, Kuulasmaa K, Giampaoli S, Mahonen M, Ngu Blackett K, et al. World Health Organization definition of myocardial infarction: 2008-09 revision. Int J Epidemiol 2011;40:139-46.

17. Zarei S, Bigizadeh S, Pourahmadi M, Ghobadifar MA. Chronic pain and its determinants: a population-based study in Southern Iran. Korean J Pain 2012;25:245-53.

18. Lopez-Marure R, Contreras PG, Dillon JS. Effects of dehydroepiandrosterone on proliferation, migration, and death of breast cancer cells. Eur J Pharmacol 2011;660:268-74.

19. Heinonen PK, Koivula T, Pystynen P. Decreased serum level of dehydroepiandrosterone sulfate in postmenopausal women with ovarian cancer. Gynecol Obstet Invest 1987;23:271-4.

20. World Health Organization. Fact sheet: the top 10 causes of death [Internet]. Geneva: World Health Organization; 2011 [cited $2013 \mathrm{Feb} 6$ ]. Available from: http://who.int/mediacentre/factsheets/fs310/en/. 
21. Mitchell LE, Sprecher DL, Borecki IB, Rice T, Laskarzewski PM, Rao DC. Evidence for an association between dehydroepiandrosterone sulfate and nonfatal, premature myocardial infarction in males. Circulation 1994;89:89-93.

22. Wang YD, Tao MF, Cheng WW, Liu XH, Wan XP, Cui K. Dehydroepiandrosterone indirectly inhibits human osteoclastic resorption via activating osteoblastic viability by the MAPK pathway. Chin Med J (Engl) 2012;125:1230-5.

23. Chen AH, Li HY, Wu VC, Lin YH, Huang TS; (and the Taiwan Primary Aldosteronism Investigation (TAIPAI) Study Group). Serum dehydroepiandrosterone sulfate concentration is lower in women with primary aldosteronism. J Renin Angiotensin Aldosterone Syst 2013 Apr 9 [Epub].

24. Aldred S, Mecocci P. Decreased dehydroepiandrosterone (DHEA) and dehydroepiandrosterone sulfate (DHEAS) concentrations in plasma of Alzheimer's disease (AD) patients. Arch Gerontol Geriatr 2010;51:e16-8.

25. Bacsi K, Kosa J, Lazary A, Horvath H, Balla B, Lakatos P, et al. Significance of dehydroepiandrosterone and dehydroepiandrosterone sulfate in different diseases. Orv Hetil 2007;148:651-7.

26. Blum CA, Mueller C, Schuetz P, Fluri F, Trummler M, Mueller B, et al. Prognostic value of dehydroepiandrosterone-sulfate and other parameters of adrenal function in acute ischemic stroke. PLoS One 2013;8:e63224.

27. Jimenez MC, Sun Q, Schurks M, Chiuve S, Hu FB, Manson JE, et al. Low dehydroepiandrosterone sulfate is associated with increased risk of ischemic stroke among women. Stroke 2013;44:1784-9.

28. Barrett-Connor E, Goodman-Gruen D. Dehydroepiandrosterone sulfate does not predict cardiovascular death in postmenopausal women. The Rancho Bernardo Study. Circulation 1995;91:1757-60.

29. Rexrode KM, Manson JE, Lee IM, Ridker PM, Sluss PM, Cook NR, et al. Sex hormone levels and risk of cardiovascular events in postmenopausal women. Circulation 2003;108:1688-93.

30. Ghebre MA, Hart DJ, Hakim AJ, Kato BS, Thompson V, Arden NK, et al. Association between DHEAS and bone loss in postmenopausal women: a 15-year longitudinal population-based study. Calcif Tissue Int 2011;89:295-302. 